\title{
MORFOANATOMÍA EN CRANICHIDEAE (ORCHIDACEAE) DE LA ESTACIÓN LOMA REDONDA DEL PARQUE NACIONAL "SIERRA NEVADA", MÉRIDA, VENEZUELA
}

\author{
Blanca A. Dugarte Corredor ${ }^{1,2} \&$ Rebeca Luque Arias $^{1}$ \\ ${ }^{1}$ Centro Jardín Botánico de Mérida, Facultad de Ciencias. Universidad de los Andes, Mérida, \\ estado Mérida, 5101, Venezuela \\ ${ }^{2}$ Autor para correspondencia: blancadu@ula.ve
}

\begin{abstract}
Resumen. Se realizó un estudio morfoanatómico de los órganos vegetativos y del pedúnculo de tres representantes de la subtribu Cranichidinae s.l. (Orchidaceae): Aa paleacea, Myrosmodes paludosa y Pterichis multiflora, de la estación Loma Redonda del Teleférico de Mérida, del Parque Nacional Sierra Nevada. Estas tres especies se estudiaron con el fin de determinar la posible existencia de caracteres diagnósticos así como la presencia de caracteres adaptativos al páramo. Se recolectaron plantas adultas. Se determinaron caracteres comunes: raíces rollizas, velamen y exodermis uniestratificado, exodermis con engrosamientos escalareiformes en las paredes radiales, espirantosomas en la corteza y hojas glabras arrosetadas. Se reporta por primera vez mesófilo diferenciado en Cranichideae. Se determinaron los siguientes caracteres diagnósticos: grosor de la corteza radicular; número de corridas de células de la empalizada y esponjoso; diferentes tipos de estelas en el pedúnculo. Algunos de estos caracteres se consideran adaptativos al ambiente: raíces rollizas, corteza compacta; hojas pequeñas y brillantes de cutícula gruesa, desarrollo de empalizada; pedúnculo con epidermis y cutícula gruesa Se considera Myrosmodes paludosa como la más xeromórfica. Se presenta una clave de caracteres morfológicos y otra de caracteres anatómicos.
\end{abstract}

ABSTRACT. A morpho-anatomic study of vegetative organs and peduncle was conducted in three representatives of the subtribe Cranichidinae s.l. (Orchidaceae): Aa paleacea, Myrosmodes paludosa, Pterichis multiflora of the Sierra Nevada National Park in Merida, Venezuela at Loma Redonda Cable car station. These three species were studied with the aim of determining possible diagnostic characters and adaptations to the paramo. Adult plants were collected. Shared characters were determined: gross root, monostratified velamen and exodermis, exodermis scalariform with thickenings in radial walls, spiranthosomes in the cortex and glabrous clustered leaves. Differentiated mesophyll is reported for the first time in Cranichideae. The diagnostic characters determined were: thickness of cortex root; number of runs of the palisade and of the spongy; in the peduncle different types of steles. Some of following characters are considered adaptive to the environment: gross root, compact cortex, small shiny leaves of thick cuticle, development of palisade; peduncle with cuticle and thick epidermis. Myrosmodes paludosa was considered the most xeromorphic species. A key using morphological and anatomical characters is presented.

Palabras clave / Key words: Aa paleacea, Cranichideae, Myrosmodes paludosa, Orchidaceae, Pterichis multiflora, Venezuela

Introducción. La tribu Cranichideae Lindl. Galeottiellinae, Goodyerinae, Manniellinae, Prescotrepresenta al grupo de las orquideas terrestres, tiinae, Pterostylidinae and Spiranthinae (Dressler confinadas mayormente a las regiones tropicales y 1993) con aproximadamente 210 especies en 17 subtropicales. Algunos de sus representantes tienen géneros (Pridgeon et al. 2003) y una distribución raíces carnosas, hojas reunidas en una roseta basal y cosmopólita (Cribb 2003). En Suramérica tienen flores muy pequeñas (Dressler 1993). Contiene nueve una distribución restringida a países andinos como subtribus: Achlydosinae, Chloraeinae, Cranichidinae, Ecuador, Perú, Colombia y Venezuela, mientras que 
en Centroamérica se han reportado representantes de los géneros $A a$ (Costa Rica) (Trujillo \& Delgado 2011) y Pterichis (Costa Rica y Jamaica) (Dueñas \& Fernández-Alonso 2009).

Los géneros Aa Rchb.f. y Myrosmodes Rchb.f. están dentro de la subtribu Prescottiinae y el género Pterichis Lindl. dentro de la subtribu Cranichidinae (Dressler 1993). Ambas subtribus comparten ciertas características que las diferencian de las demás, como es el caso de las flores no-resupinadas, y se distinguen entre ellas porque las Prescottiinae tienen velamen tipo Spiranthes mientras que las Cranichidinae tienen velamen tipo Calanthe (Salazar et al. 2009).

Figueroa et al. (2008), estudiando engrosamiento de las paredes secundarias de las células del velamen, tipo de tilosomas y espacios supraendodermales marcaron que forman un grupo monofilético. Sin embargo este grupo es muy diverso y algunos representantes no han sido incluidos en ellos por lo que se requieren más estudios para verificar si la ecología tiene un papel fundamental en sus estructuras (Salazar et al. 2009). Estudios realizados por Salazar et al. (2003) muestran un análisis filogenético, donde la subtribu Prescottinae es parafilética y consiste en dos clados altamente apoyados: "las Prescottinae de elevaciones altas" conformada por los géneros $A a$, Altensteinia, Gomphichis, Myrosmodes, Porphyrostachys y Stenoptera y "las Prescottinae de elevaciones bajas" conformada por los géneros Prescottia y Pseudocranichis (Álvarez 2005).

Venezuela es uno de los países más ricos en especies de orquídeas. El Nuevo Catálogo de la Flora Vascular de Venezuela (2009) reporta 213 géneros y 1506 especies. Alrededor de 283 especies (+1 subespecie) son endémicas. El país presenta ambientes variados como son tierras áridas, bosques en todos los pisos altitudinales, sabanas, páramos y otros (Lasser 1969).

Las características morfológicas de las plantas del páramo son por lo general de hábito arrosetado con raíces gruesas para almacenaje, desarrollan hojas pequeñas y hacen modificaciones para aumentar la reflexión de la luz (Eames \& MacDaniels 194, Fahn 1974, Dahlgren 1985, Lindorf et al. 1991, Escalona 1999, Rojas 2000). Anatómicamente, las plantas desarrollan una epidermis de paredes engrosadas, cutículas engrosadas y tejidos compactos (Pyykkö
1966, Rada et al. 1985, Lindorf et al. 1991, Roth 1990, Luque et al. 1999).

El presente estudio pretende evaluar las características morfoanatómicas de Aa paleacea, Myrosmodes paludosa (subtribu Prescottiinae) y Pterichis multiflora (subtribu Cranichideae) con el fin de determinar las semejanzas y diferencias entre ellas y la presencia de caracteres que indiquen estrategias adaptativas al medio ambiente páramo. Este trabajo aportará datos importantes que pueden ser tomados en cuenta para el estudio de estos grupos.

\section{Materiales y métodos}

ÁREA DE ESTUdio - La estación Loma Redonda del teleférico de Mérida se encuentra en el Parque Nacional Sierra Nevada, al sureste de la ciudad de MéridaVenezuela, entre los $8^{\circ} 34^{\prime}$ y $8^{\circ} 36^{\prime} \mathrm{N}$ y $71^{\circ} 4^{\prime}$ y $71^{\circ} 7^{\prime} \mathrm{W}$ y a unos $4045 \mathrm{~m}$ de elevación (Petit 1984), con una temperatura media anual de $3,0{ }^{\circ} \mathrm{C}$ una precipitación media anual de $1.553 \mathrm{~mm}$ y con un régimen unimodal tropical (Monasterio \& Reyes 1980). Pertenece al núcleo central de los Andes venezolanos y en la zona se observan caracteres ambientales estresantes como cambios bruscos de temperatura, humedad, hielo y deshielo, radiaciones altas y bajas, estrés hídrico y fuertes vientos casi constantes durante todo el año.

Se estudiaron dos representantes de la subtribu Prescottiinae: Aa paleacea y Myrosmodes paludosa y un representante de la subtribu Cranichidinae: Perichis multiflora (Tabla 1).

Se identificó el material colectado utilizando las claves de Garay (1978). Para los estudios anatómicos se utilizaron hojas completamente expandidas, raíces y pedúnculo de plantas adultas en floración y fijados en una solución de formaldeído 37\%, ácido acético glacial y ethanol 70\% (FAA) (Johansen 1940). Se realizaron cortes transversales a mano alzada en la región media de todos los órganos. Para separar la epidermis del mesófilo se utilizó la técnica de Jeffrey citada por Johansen (1940), tanto los cortes como las epidermis fueron coloreados con la doble tinción de azul de astra 0,5\% y fuchsina básica 0,2\% según Luque et al (1996) y fueron montados en glicerina 50\% (Johansen 1940). El tamaño de las células de los diferentes tejidos en cada órgano se obtuvieron midiendo largo por ancho de 10 células en tres muestras diferentes y se promediaron los valores obtenidos. Para la densidad estomática 
TABla 1. Lista de las especies estudiadas de Cranichideae $s . l$.

\begin{tabular}{l|l|l|l} 
Taxón & Testigo & Herbario & Zona de colecta \\
\hline Aa paleacea (Kunth) Rchb. f. & $\begin{array}{l}\text { Estrada et al. 1027a } \\
\text { Dugarte \& Contreras 102 } \\
\text { Dugarte \& Contreras, 106 } \\
\text { Dugarte \& Contreras 107 }\end{array}$ & $\begin{array}{l}\text { Al oeste de la estación dentro del bosque } \\
\text { de Polylepis sericeae. Suelos con } \\
\text { abundante humus }\end{array}$ \\
\hline Myrosmodes paludosa (Rchb. f.) Garay & $\begin{array}{l}\text { Estrada et al. 1023 } \\
\text { Dugarte \& Contreras 100 } \\
\text { Dugarte \& Contreras 101 } \\
\text { Dugarte \& Contreras 103 } \\
\text { Dugarte \& Contreras 104 }\end{array}$ & MERC & $\begin{array}{l}\text { Al oeste de la estación hacia y por los } \\
\text { alrededores del bosque de Polylepis } \\
\text { sericeae. Suelos pedregosos y } \\
\text { encharcados }\end{array}$ \\
\hline Pterichis multiflora (Lindl.) Schltr. & $\begin{array}{l}\text { León \& Gaviria 491 } \\
\text { Estrada et al. 1027b }\end{array}$ & MERC & $\begin{array}{l}\text { Al oeste de la estación dentro del bosque } \\
\text { de Polylepis sericeae. Suelos con } \\
\text { abundante humus }\end{array}$ \\
\hline
\end{tabular}

(DE) se utilizó la formula de Salisbury (1927) según Wilkinson (1979) y para el índice estomático (IE) se utilizó la fórmula sugerida por Wilkinson (1979). Los datos utilizados fueron valores promedios obtenidos en tres muestras diferentes. Los cortes anatómicos fueron observados en un microscopio ZEISS AXIOSCOP 20 y se tomaron micrografías con una cámara MC80 adosada al microscopio.

Los datos cuantitativos de las características anatómicas son mostrados en las tablas 2,3 y 4 . Se presenta así mismo una clave de caracteres morfológicos y otra de caracteres anatómicos que será útil para cuando no se tenga la oportunidad de colectar con flores.

\section{Resultados}

MoRfologíA - Para la caracterización morfológica se describieron la raíz, hoja y pedúnculo de cada especie y para el tamaño se midieron los órganos de cada indivíduo y se promediaron los valores obtenidos.

Aa paleacea: hierbas terrestres de 35,0-60,0 cm de alto (Figs. 1A, 1B), crece en suelos pedregosos o en suelos con hojarasca y humus, solitarias o en grupo; raíces rollizas y suculentas de 4,0-7,5 cm de largo por 0,47 $\mathrm{cm}$ de diámetro; hojas envainadoras, dispuestas en rosetas (Fig. 1A), lanceolada hasta elíptico-lanceolada, glabras, de superficie opaca de unos 6,0-15,0 cm de largo y 1,4-1,6 de ancho. Pedúnculo lateral erecto de unos 18,0-42,0 cm de largo por 0,3 cm de diámetro, revestido por numerosas bracteas (Fig. 1B; br); espiga densa de 4,0-8,0 cm de largo de flores blancas pequeñas glabras no-resupinadas (Fig. 1A, 1B). Sépalo dorsal y pétalos libres de la columna. Labio calceolado con margen fimbriado involuto.
Myrosmodes paludosa: hierbas terrestres de 2,0-12,0 $\mathrm{cm}$ de alto (Fig. 1C-1E), crecen en suelos pedregosos o húmedos, solitarias o en grupo (Fig. 1C, 1D); raíces rollizas y suculentas de 4,5-8,0 cm de largo por $0,4 \mathrm{~cm}$ de diámetro (Fig. 1E); hojas envainadoras, dispuestas en rosetas (Fig. 1C), elíptico-oblanceolada hasta aovada, glabras, de superficie brillante de 4,0-12,5 $\mathrm{cm}$ de largo y 1,4-1,6 de ancho. Pedúnculo lateral erecto de 2,0-5,0 cm de largo por 0,44 $\mathrm{cm}$ de diámetro, revestido por brácteas (Fig. 1E; br), con entrenudos acortados solapándose las brácteas; espiga densa de 2,0-5,0 cm de largo de flores blancas pequeñas glabras no-resupinadas (Figs. 1C-1E). Sépalo dorsal y pétalos adnados al dorso de la base de la columna. Labio tubular con margen fimbriado nunca involuto.

Pterichis multiflora: hierbas terrestres de 20,0-48,0 cm de alto (Fig. 1F, 1G), crecen en suelos con hojarasca y humus, solitarias (Fig. G); raíces rollizas y suculentas de 3,0-7,5 cm de largo por 0,38 cm de diámetro; hojas pecioladas, dispuestas en rosetas (Fig. G), oblongos u oblongo-lanceolada, glabras, de superficie opaca de unos 4,0-15,5 cm de largo y 2,5-2,8 cm de ancho. Pedúnculo terminal erecto de aproximadamente 30,0 $\mathrm{cm}$ de largo por $0,32 \mathrm{~cm}$ de diámetro glabro en la base y piloso el resto, revestido por bracteas foliosas (Fig. $1 \mathrm{~F}$; br); espiga subdensa de 5,0-10,0 cm de largo de flores amarillas pilosas con rayas rojas no-resupinadas (Fig. 1F, 1G). Sépalos semejantes libres, el dorsal péndulo y los laterales erectos (Fig. 1F, 1G); pétalos más angostos que el sépalo dorsal. Labio súpero y algo adnado a la base de la columna.

Se pudo observar en campo que M. paludosa tiene un período de crecimiento vegetativo mayor, esto es 


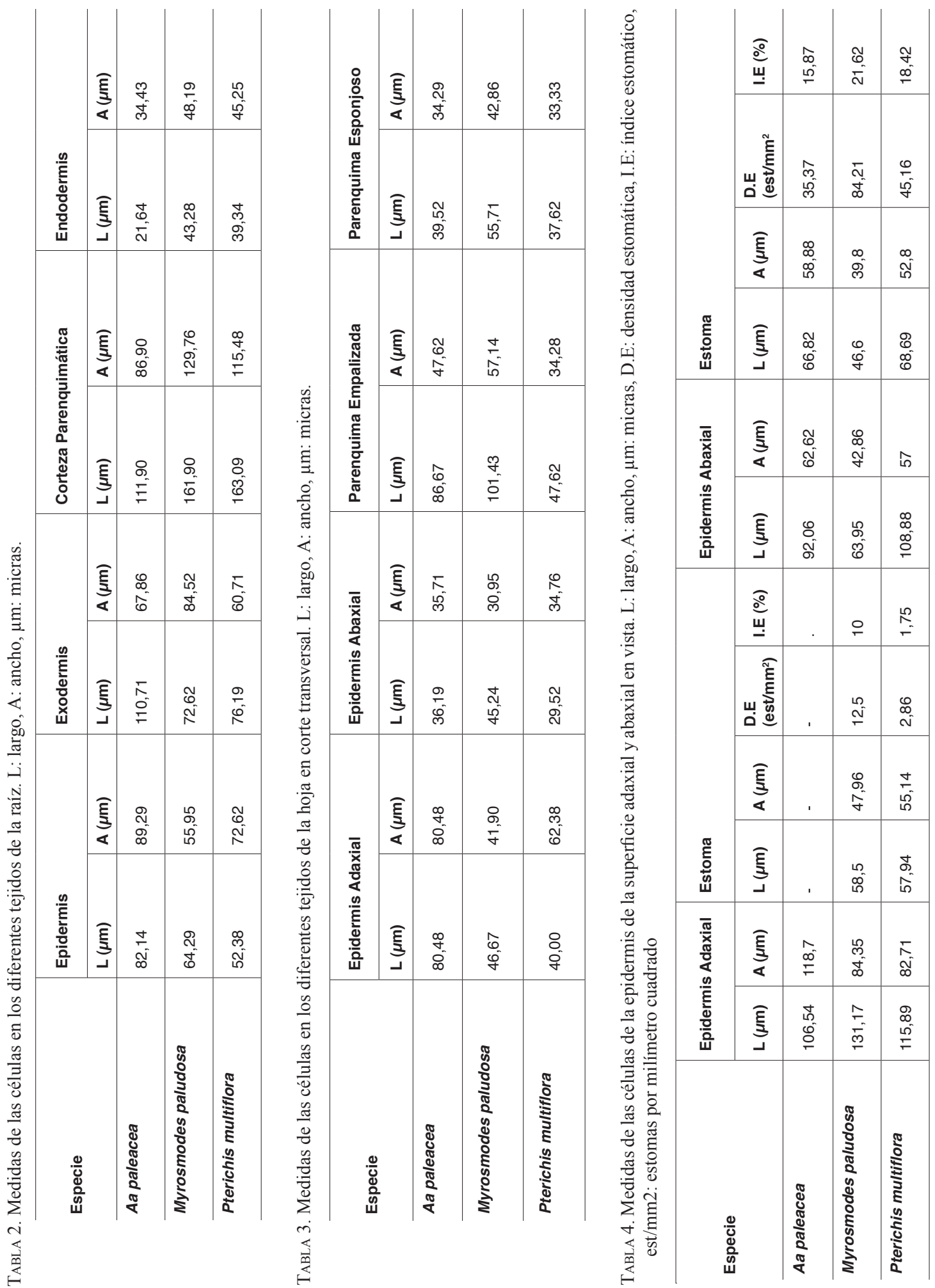



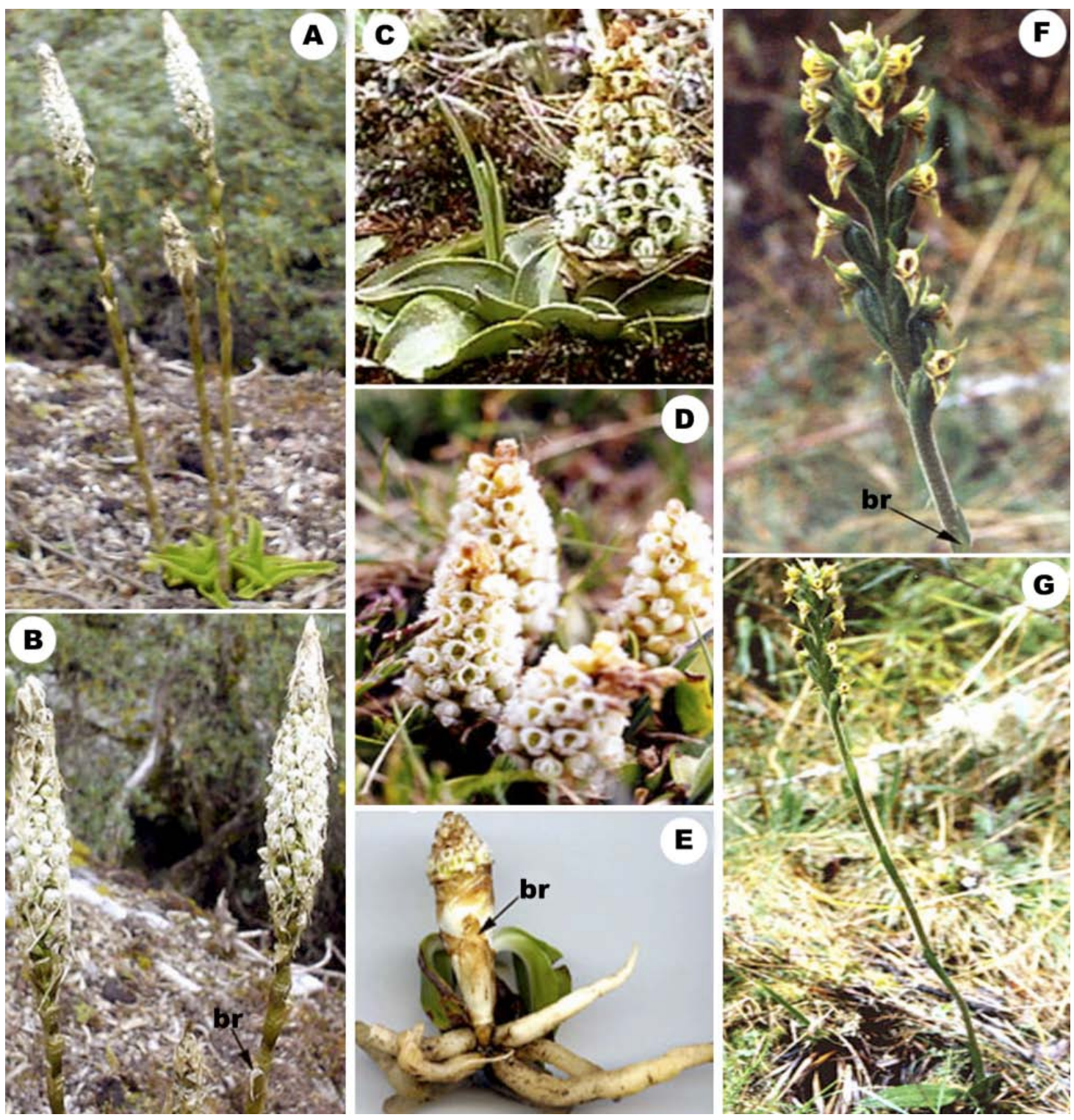

Figura 1. Características morfológicas. A, B: Aa paleacea. C-E: Myrosmodes paludosa. F, G: Pterichis multiflora. (br= bractea). Fotografías por B. Dugarte Corredor (A-E) y J. Estrada (F, G).

porque las plantas se pueden conseguir solo en estado vegetativo fuera de la época de floración, mientras que A. paleacea y $P$. multiflora no se encuentran en estado vegetativo fuera de la época de floración.

Anatomía - Aa paleacea: en corte transversal se evidencia la raíz con velamen de una corrida de células (Fig. 2A, 2B; Ve) con pelos radicales (Fig. 2A; PR); interna al velamen se observa la exodermis formada por una corrida de células (Fig. 2A, 2B; Ex), con las paredes periclinal externa y radial engrosadas, en las paredes radiales se observa un engrosamiento escalareiforme (Fig. 2B; flecha). Corteza parenquimática, compacta de 13 a 14 corrida de células (Fig. 2A, 2C, 2D; P) observándose los amiloplastos (Fig. 2C; am) con uno a cuatro granos de almidón (Fig. 2C; al). Cilindro central rodeado por la endodermis (Fig. 2D; En) con bandas de Caspary (Fig. 


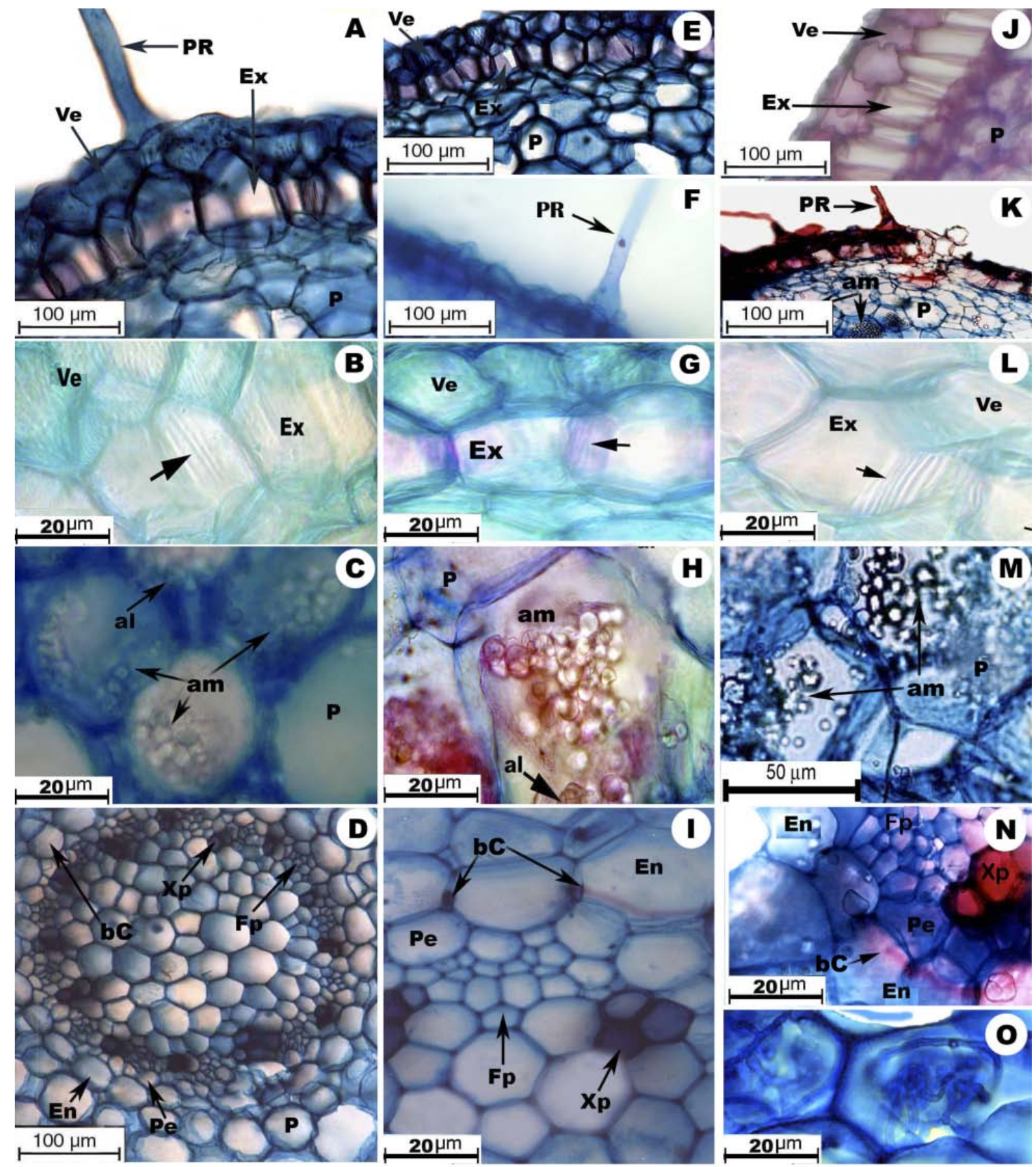

Figura 2. Corte transversal de la raíz. A-D: Aa paleacea. E-I: Myrosmodes paludosa. J-O: Pterichis multiflora. (Ve= velamen; $\mathrm{PR}=$ pelo radical; $\mathrm{Ex}=$ exodermis; $\mathrm{P}=$ parénquima; $\mathrm{am}=$ amiloplasto; $\mathrm{al}=$ almidón; $\mathrm{En}=\mathrm{endodermis} ; \mathrm{bC}=$ bandas de Caspary; $\mathrm{Pe}=$ periciclo; $\mathrm{Xp}=$ xilema primario; $\mathrm{Fl}=$ floema).

2D; bC) interna a ella el periciclo de una corrida de células (Fig. 2D; Pe) y se observa la estela poliarca con 10-16 polos del xilema (Fig. 2D; Xp) alternando con el floema primario (Fig. 2D; Fl). El corte transversal de hoja muestra: hoja bifacial hipostomática, epidermis de la superficie adaxial (Fig. 3A, 3B; Ead) con las paredes periclinales externas papilosas (Fig. 3A, 3B, $4 \mathrm{~A} ; \mathrm{Pl}$ ), cubierta por una cutícula más o menos gruesa y estriada (Fig. 3B, 4A; Cut), paredes periclinales internas un poco cóncavas. En vista paradermal se 


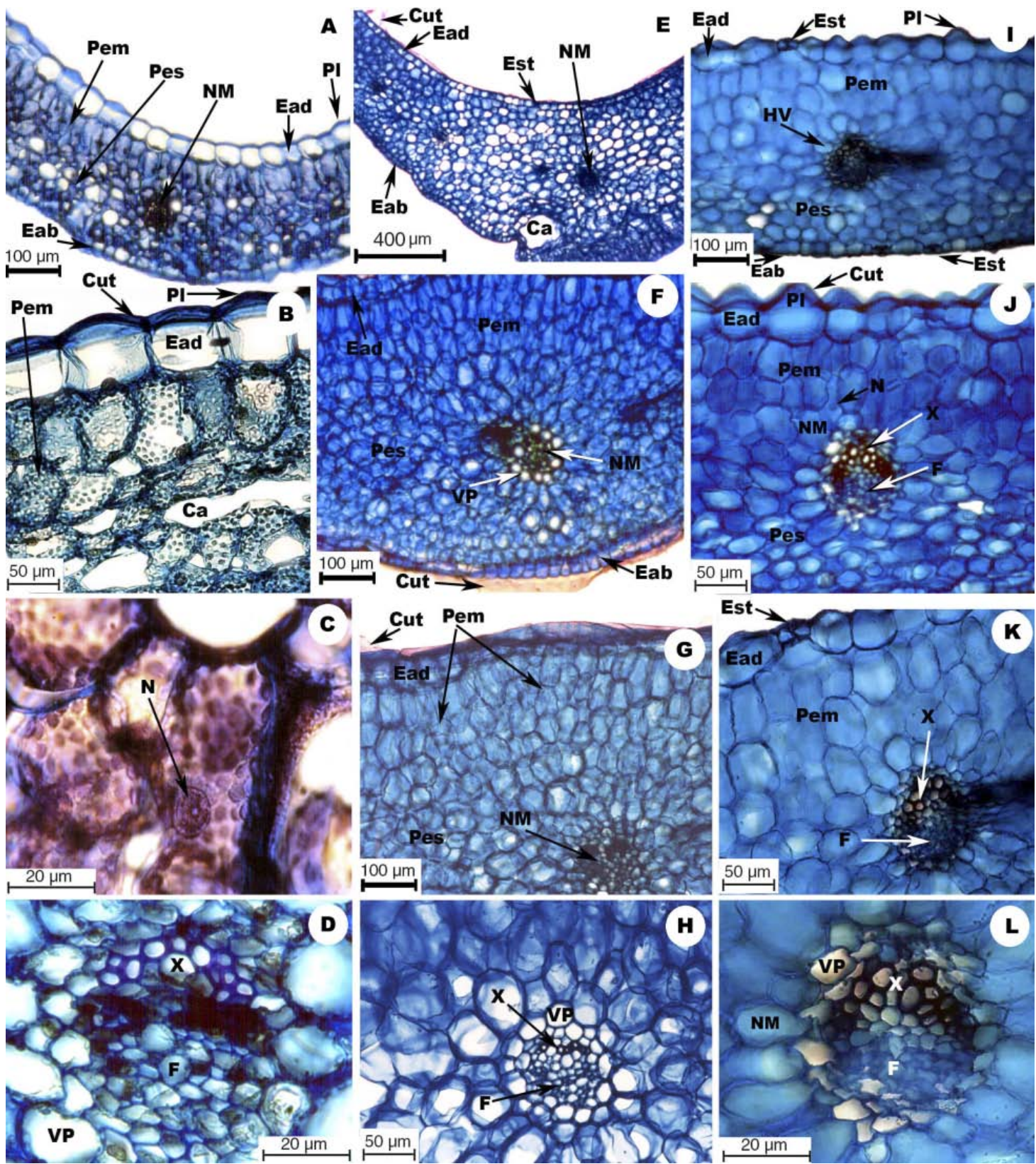

Figura 3. Corte transversal de la hoja. A-D: Aa paleacea. E-H: Myrosmodes paludosa. I-L: Pterichis multiflora. (Ead = epidermis de la superficie adaxial; Eab = epidermis de la superficie abaxial; Cut = cutícula; Pem = parénquima en empalizada; $\mathrm{Pes}=$ parénquima esponjoso; $\mathrm{NM}=$ nervio medio; $\mathrm{Ca}=$ cámara aerífera; $\mathrm{N}=$ núcleo; $\mathrm{VP}=\mathrm{vaina}$ parenquimática; $\mathrm{X}=$ xilema; $\mathrm{Fl}=$ floema).

observan células poliédricas de 5 a 7 caras, de paredes más o menos gruesas y rectas (Fig. 4A). Epidermis de la superficie abaxial, en corte transversal, formada por células cuadradas (Fig. 3A; Eab). En vista paradermal se observa formada por células 4-5-6 caras de paredes más o menos gruesas, de paredes sinuosas (Fig. 4B); estomas anomocíticos rodeados por 3-4 (5) células anexas (Fig. 4B; Est). Densidad estomática de 45,12 est $/ \mathrm{mm}^{2}$, índice estomático de 14,07\%. Mesófilo diferenciado, compacto; parénquima en empalizada 
(Fig. 3A. 3B; Pem) formado por 1-2 corridas de células cortas; parénquima esponjoso de 6 corridas de células (Figs. 3A, 3B; Pes), se observan cámaras aeríferas hacia la superficie abaxial (Fig. 3B; Ca). Es notable en todo el mesófilo que las células poseen un núcleo grande con nucleolo (Fig. 3C; N) y están llenas de cloroplastos (Fig. 3B, 3C). Haces vasculares colaterales cerrados (Fig. 3D) rodeados por una vaina parenquimática simple (Fig. 3D; VP) con almidón. El pedúnculo en corte transversal se observa de contorno elíptico, con epidermis de una corrida de células (Fig. 5B; Ep). Cutícula gruesa (Fig. 5B; Cut). Corteza parenquimática muy reducida de 1 a 2 corridas de células (Fig. 5B; P) seguida por un anillo de 5 a 6 corridas de células esclerenquimáticas, (Fig. 5B; Es). Internamente se observa la atactoestela con haces vasculares colaterales de mayor tamaño (Figs. 5A y B; HV) rodeados de una vaina parenquimática simple, los menores formados por xilema solamente. Se observa lisis de la zona central (Fig. 5A; *).

Myrosmodes paludosa: en corte transversal se evidencia la raíz con velamen de una corrida de células (Fig. 2E, 2G; Ve) con pelos radicales (Fig. 2F; PR); interna al velamen se observa la exodermis formada por una corrida de células (Fig. 2E, 2G; Ex), con las paredes periclinal externa y radial engrosadas, en las paredes radiales se observa un engrosamiento escalareiforme (Fig. 2G; flecha). Corteza parenquimática, compacta de 16 a 18 corridas de células (Fig. 2E, 2H; P), observándose los amiloplastos (Fig. 2H; am) con uno a cuatro granos de almidón (Fig. 2H; al). En la figura 2I se observa el cilindro central rodeado por la endodermis (Fig. 2I; En) con bandas de Caspary (Fig. 2I; bC) seguida internamente el periciclo con una corrida de células (Fig. 2I; Pe) y se observa la estela poliarca con aproximadamente 11 polos xilemáticos (Fig. 2I; $\mathrm{Xp}$ ) alternando con el floema primario (Fig. 2I; Fl). El corte transversal de hoja muestra: hoja bifacial anfiestomática, epidermis de la superficie adaxial (Fig. 3E-3G; Ead) formada por células de paredes engrosadas más o menos isodiamétricas, con las paredes periclinales internas un poco cóncavas, cubierta por una cutícula lisa y gruesa (Fig. 3E, 3G; Cut). En vista paradermal se observan células poliédricas de 5 a 6 caras, de paredes muy gruesas y rectas (Fig. 4C). Estomas anomocíticos y diacíticos rodeados por 2-3-4 (5) células anexas (Fig. 4C; Est). Densidad estomática de $17,65 \mathrm{est} / \mathrm{mm}^{2}$, índice estomático de $8,85 \%$. Epidermis de la superficie abaxial, en corte transversal, formada por células más o menos isodiamétricas (Fig. 3E, 3F; Eab) cubierta por una cutícula más o menos gruesa (Fig. 3F; Cut). En vista paradermal se observan células de 4-5-6 caras de paredes gruesas, rectas a ligeramente sinuosas (Fig. 4D); estomas anomocíticos rodeados por 3-4-5 células anexas (Fig. 4D; Est). Densidad estomática de 41,18 est $/ \mathrm{mm} 2$, índice estomático de $19,87 \%$. En vista paradermal en las células epidérmicas, se puede observar claramente el núcleo (Fig. 4C, 4D; N). Mesófilo poco diferenciado, compacto; parénquima empalizada (Fig. 3F, 3G; Pem) formado por 4-5 corridas de células cortas; parénquima esponjoso de 5-6-7 (8) corridas de células (Fig. 3F, 3G; Pes). Nervio Medio (Fig. 3E-3G; NM) formado por un haz colateral. Haces vasculares colaterales cerrados rodeados por una vaina parenquimática simple (Fig. 3H; VP) con almidón, se observa cámara aerífera hacia la superficie abaxial (Fig. 3E; Ca). El pedúnculo en corte transversal se observa de contorno elíptico, con epidermis de una corrida de células (Fig. 5C; Ep). Cutícula gruesa (Fig. 5C; Cut). Corteza parenquimática de 7 a 8 corridas de células (Fig. 5C; P) seguida por un anillo de 3 corridas de células esclerenquimáticas (Fig. 5C; Es). Internamente se observa la estela formando anillos concéntricos con haces vasculares colaterales (Fig. 5C; HV), haces del anillo más externo se observan relacionados con el tejido esclerenquimático. En la Figura 5D se observa con más detalle el xilema (Fig. 5D; X) y el floema (Fig. 5D; Fl) rodeados por una vaina parenquimática simple en cuyas células se observan granos de almidón (Fig. 5D; al). Se observa médula parenquimática compacta (Fig. $5 \mathrm{C} ; \mathrm{M}$ ) y muy notable el núcleo en cada célula (Fig. 5C; N).

Pterichis multiflora: en corte transversal se evidencia la raíz con velamen de una corrida de células (Figs. 2J y L; Ve) con pelos radicales (Fig. 2K; PR); interna al velamen se observa la exodermis formada por una corrida de células (Figs. 2J y L; Ex), con las paredes periclinales externa y radiales engrosadas, en las paredes radiales se 

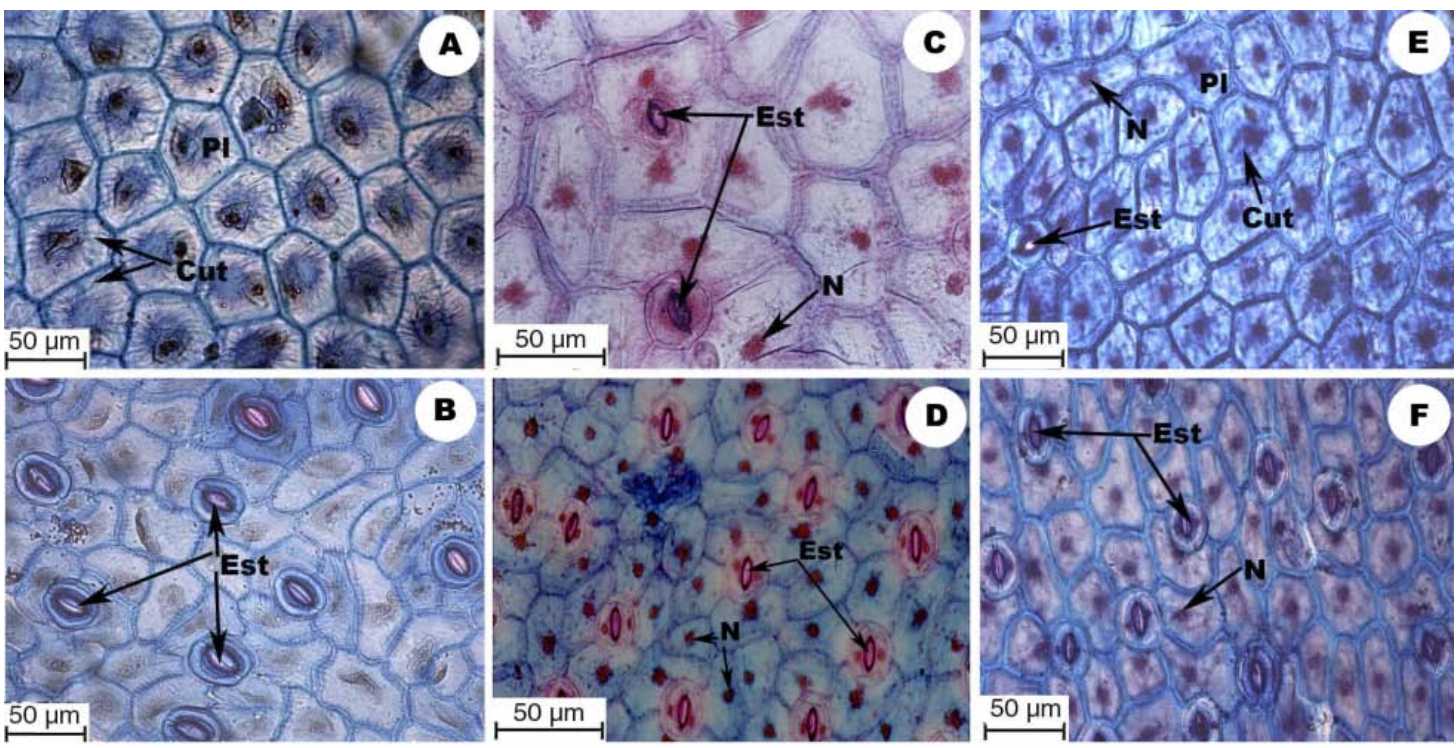

Figura 4.- Vista paradermal de la epidermis. A,C,F: Epidermis adaxial. B,D,G: Epidermis abaxial. Aa paleacea A,B. Myrosmodes paludosa CyD. Pterichis multiflora EyF. (Cut: cutícula; Est: estoma; P: papila; N: núcleo).

A,B: Aa paleacea (A: epidermis adaxial; B: epidermis abaxial). C,D: Myrosmodes paludosa (C: epidermis adaxial; D: epidermis abaxial). E,F: Pterichis multiflora (E: epidermis adaxial; F: epidermis abaxial). (Cut = cutícula; Est = estoma; $\mathrm{P}=$ papila; $\mathrm{N}=$ núcleo).

observa un engrosamiento escalareiforme (Fig. 2L; flecha). Corteza parenquimática, compacta de 13 a 15 corrida de células (Figs. 2J y M; P) observándose los amiloplastos (Fig. 2K y M; am). Es notoria la presencia de hifas (Fig. 2O; Hf). Se puede observar el cilindro central rodeado por la endodermis (Fig. 2N; En) con bandas de Caspary (Fig. 2N; bC), interna a ella el periciclo de una corrida de células (Fig. 2N; Pe). Estela con 8 polos xilemáticos (Fig. 2N; Xp) alternando con el floema primario (Fig. $2 \mathrm{~N}$; Fl). El corte transversal de hoja muestra: hoja bifacial anfiestomática, epidermis de la superficie adaxial (Fig. 3I-3K; Ead) formada por células rectangulares con las paredes periclinales externas papilosas (Fig. 3I , 3J; P1), las internas un poco cóncavas, cubierta por una cutícula gruesa y estriada (Fig. 3J; Cut). En vista paradermal se observan células de 5 a 6 (7) caras de paredes gruesas, rectas a sinuosas (Fig. 4E) estomas anomocíticos rodeados por 4-5 células anexas (Fig. 4E; Est). Densidad estomática de 15,71 est $/ \mathrm{mm}^{2}$, índice estomático de $6,62 \%$. Epidermis de la superficie abaxial, en corte transversal, formada por células rectangulares (Fig. 3I; Eab). En vista paradermal se observan células de 5 a 6 (7) caras de paredes muy gruesas, rectas a sinuosas (Fig. 4F).
Estomas anomocíticos rodeados por (3) 4-5 células anexas (Fig. 4F; Est). Densidad estomática de 68,65 est $/ \mathrm{mm} 2$, índice estomático de $24,14 \%$. En vista paradermal en las células epidérmicas, se puede observar claramente el núcleo (Fig. 4E, 4F; N). Mesófilo poco diferenciado, compacto; parénquima en empalizada (Fig. 3I-3K; Pem) formada por 2 corridas de células cortas; parénquima esponjoso de 7-8 corridas de células (Fig. 3I, 3J; Pes). Se puede notar algunas células con su núcleo (Fig. 3J; N). Nervio Medio (Fig. 3J, 3L; NM) formado por un haz colateral. Haces vasculares colaterales cerrados (Fig. 3I; HV) rodeados por una vaina parenquimática simple (Fig. 3L; VP) con almidón. El pedúnculo en corte transversal se observa de contorno elíptico, con epidermis de una corrida de células (Fig. 5F; Ep) con tricomas pluricelulares (Fig. 5F, 5G; Tri). Cutícula gruesa (Fig. 5F; Cut). Corteza parenquimática de 3 a 4 corridas de células (Fig. 5F; P) seguida por un anillo de 5 a 6 corridas de células esclerenquimáticas (Fig. 5F; Es). Internamente se observa la atactoestela con haces vasculares colaterales rodeados de una vaina parenquimática simple (Fig. 5E; HV). Se observa lisis en la zona central (Fig. 5E; *). 

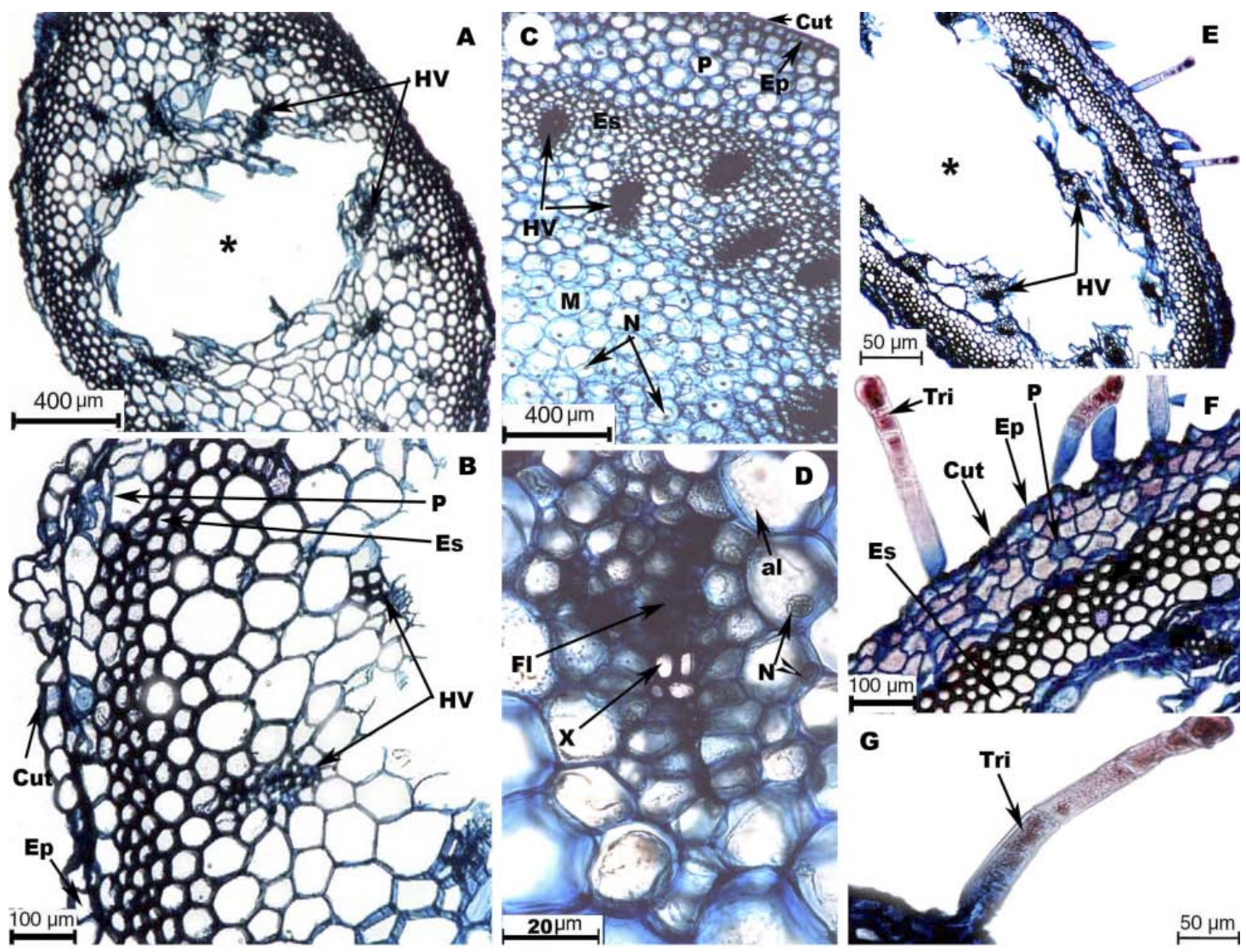

Figura 5. Corte transversal del Pedúnculo. A,B: Aa paleacea. C,D: Myrosmodes paludosa. E,G: Pterichis multiflora. (Ep = epidermis; cut = cutícula; $\mathrm{P}=$ corteza parenquimática; $\mathrm{Es}=$ células esclerenquimáticas; $\mathrm{HV}=$ haces colaterales; $\mathrm{M}=$ médula; $\mathrm{N}=$ Núcleo; al = almidón; * = lisis medular; Tri = tricoma).

\section{Discusión}

Las especies estudiadas son plantas terrestres caracterizadas por tener hojas en rosetas basales y raíces gruesas de almacenaje, características representativas de la tribu Cranichideae (Dahlgren 1985). Las raíces rollizas y suculentas, son órganos de reserva apropiados para que estas plantas puedan vivir en las condiciones del páramo y soportar los cambios estacionales.

El velamen observado es de tipo spiranthes y coincide con los tipos de velamen propuestos por Porembski y Barthlott (1988) así como también una de las característica de los representantes de la tribu Prescottiinae como A. palacea y $M$. paludosa, no obstante el velamen que muestra $P$. multiflora difiere con lo descrito por Salazar (2009) donde indica que el velamen para los representantes de la tribu Cranichidinae a la cual está incluido este género es de tipo Calanthe.
La exodermis además del engrosamiento de las células en las paredes periclinal externa, muestra un engrosamiento escalareiforme en las paredes radiales. En un estudio comparativo de las tribus Cranichidae, Diceratosteleae y Tropidieae realizado por Stern et al. (1993b) este tipo de engrosamiento fue encontrado sólo en especies de la tribu Cranichideae, estos autores suponen que funcionan como estructuras de rigidez que previenen el colapso de las células exodérmicas. Solereder \& Meyer (1930) (según: Stern et al. 1993b) describieron este tipo de engrosamiento, pero no lo ilustraron ni lo comentaron. Aunque no se trata exactamente de deposición de bandas de Caspary, podríamos considerar que este engrosamiento también puede estar actuando en el control del flujo de agua y nutrientes, regulando el flujo de los mismos en la raíz tal como lo indican (Perumalla \& Peterson 1986, Hose et al. 2001, García 2004). Este carácter fue muy 
útil para Figueroa et al. (2007) porque les permitió delimitar al clado Prescottiinae -Spiranthinae Cranichidinae.

La corteza compacta evita la acumulación de agua intercelular por lo que incrementa su resistencia a la congelación. El número de corridas de células es menor para A. paleacea, según Fanh (1967), Jenik (1978), Lindorf (1991), un número menor de corridas de células favorecería el rápido flujo de agua al interior del cilindro central. Estas especies crecen en microambientes similares, la diferencia observada en la corteza puede deberse quizás a que M. paludosa presenta un período de crecimiento vegetativo mayor.

Las micorrizas en la corteza de P. multiflora, ubicadas en las células más externas de la corteza, coincide con lo observado por Stern et al. (1993b). Algunas orquídeas en condiciones naturales germinan sólo después de establecer simbiosis con el micelio de un hongo (Dalhgreen 1985). Esta interacción ha sido considerada como un mecanismo de adaptación que ha permitido a las orquídeas mantenerse en condiciones climáticas extremas y tener una distribución a nivel mundial (Garay 1963). Para el año 1902 Noel Bernard (según Arber 1961) describió que la mayoría de las semillas de orquídeas no germinan naturalmente en ausencia de micorrizas y para la misma época Hans Buergeff (Arditti 1992) demostró que la interacción micorriza-orquídea eran bastantes específicas. Sin embargo, se conoce ahora (Rasmussen \& Whigham 1998) que algunas especies tienen interacciones específicas con ciertas especies de hongos, otras (Arditti 1992) tienen una relación general con muchas especies de hongos. Esta variedad de tipos de interacción micorrízica en orquídeas podría explicar el hecho de no haberse observado en A. paleacea ni en M. paludosa o posiblemente deba repetirse la observación con técnicas especializadas en micorrizas.

Los amiloplastos son llamados espirantosomas por Stern et al. (1993a) y fueron observadas en las subtribu Diceratosteleae, Tropidieae y Cranichideae donde el almidón es sintetizado y reservado en amiloplastos típicos; además estos espirantosomas no fueron observados en ninguna Orchidoideae ni en células de otras orquídeas que tenían disponibles para el momento de sus estudios. Los espirantosomas son cuerpos esféricos que se encuentran en el citosol, cada uno conteniendo numerosos granos diminutos de almidón que constituirían reserva de carbohidratos.

El gran desarrollo observado de las bandas de Caspary parece ser una características bastante común en las plantas que crecen en los páramos, observándose no solamente en raíz sino también en tallo y hoja (Luque 2000). Según Van Fleet (1961) el desarrollo de las bandas de Caspary está fuertemente influenciado por factores ambientales tales como la luz, temperatura, nutrición, entre otros; por lo tanto se considera que en estas especies las condiciones de cambios de temperatura diaria y exposiciones de sequías estacionales podrían estar influenciando en el desarrollo de grandes bandas de Caspary.

La diferencia que se observa entre el número de polos xilemáticos, siendo mayor en A. paleacea, seguida de M. paludosa y $P$. multiflora hace que sea más rápida y eficiente la llegada del agua y los nutrientes al xilema para su rápida conducción (Luque 2004).

En cuanto a la morfología foliar, M. paludosa presenta hojas más adosadas al suelo, más pequeñas y brillantes y la lámina más gruesa que $A$. paleacea y $P$. multiflora que presentan hoja más alargadas y $P$. multiflora es la que presenta la roseta más laxa con las hojas más separadas del suelo; $M$. paludosa crece en lugares un poco más expuestos y las características mencionadas le proporcionan rigidez, ayudan a evitar la desecación por reflexión de la luz y evitan la acción de los vientos excesivos que se da en este tipo de ambiente (Eames \& MacDaniels 1947, Fahn 1974, Dahlgren 1985, Lindorf 1991, Escalona 1999, Rojas 2000).

Anatómicamente la epidermis de paredes celulares gruesas y cutícula gruesa en ambas superficies, se ha interpretado como un mecanismo para el control de la transpiración, además sirve de protección contra la luz intensa, evitando que los tejidos internos puedan calentarse excesivamente; también ayuda a evitar los daños mecánicos producidos por el viento y previenen el colapso o marchitamiento de los tejidos de la hoja cuando hay pérdida de agua en exceso (Pyykkö 1966, Lindorf et al. 1991, Roth 1991, Ely 1996, Torres 1998, Escalona 1999, Rojas 2000).

Las células papilosas de la epidermis de la superficie adaxial en $P$. multiflora y A. paleacea, también llamadas células en forma de lente, se 
concidera que su función puede ser concentrar la luz incidente y dirigirla hacia el mesófilo asimilador subyacente (Gaulhofer 1908, Wilkinson 1979, Lindorf et al. 1991, Roth 1991). Plantas de las especies mencionadas crecen, en la Estación Loma Redonda, dentro del bosque de Polylepis, por lo tanto más protegidas, y las papilas epidérmicas favorecerían la captación y aprovechamiento de la luz.

Myrosmodes paludosa y P. multiflora presentan hojas anfiestomáticas, donde P. multiflora tiene estomas en la superficie adaxial solo ocasionales, de ahí los valores de densidad estomática e índice estomático tan bajos para esta especie en esta superficie. En la superficie abaxial presentan una baja densidad estomática lo que puede considerarse como una adaptación a las altas altitudes y al estrés hídrico, la reducción de la densidad estomática funcionaría como un mecanismo de control transpiratorio (Colmenares et al. 2005).

Presentan estomas anomocíticos, sin embargo, $M$. paludosa también presenta estomas diacíticos, según Stern et al. (1993 b), en los representantes de la tribu se pueden conseguir diferentes tipos de estomas, inclusive en la misma hoja como es el caso de M. paludosa.

Se reporta, en este trabajo, por primera vez la presencia de mesófilo diferenciado en plantas pertenecientes a las subtribus Prescottiinae y Chranichiidinae; mesófilo diferenciado y compacto, favorece el transporte más eficiente del agua (Luque et al. 1999) y además evita que se acumule agua intercelular por lo que incrementa su resistencia a la congelación (Rada et al. 1985).

El parénquima en empalizada, a pesar de estar formado por células casi isodiamétricas o cuadradas, se observa bien diferenciado del parénquima esponjoso que está formado por células redondeadas a ovaladas. Según Haberlandt (1884), la forma de las células del parénquima fotosintético puede variar desde células isodiamétricas, como las de las especies estudiadas, hasta células alargadas en sentido paralelo a la superficie de la hoja o perpendiculares a la misma y también células braciformes y en forma de embudo. Algunos autores (Pyykkö 1966, Esau 1976, Roth 1990, Lindorf et al. 1991, Dengler 1994, Ely 1996) consideran que células de la empalizada cortas, como las que presentan las especies estudiadas, son un carácter xeromórfico ya que, además de la intensidad luz, el desarrollo del parénquima en empalizada es estimulado por la disponibilidad de suficiente agua en el suelo. Estas observaciones contrastan con lo expresado por Stern et al. (1993b), en su estudio sobre anatomía comparada de los órganos vegetativos de Spiranthoideae, consideran que los representantes de la subtribu Prescottiinae a la cual pertenecen $A$. paleacea y $M$. paludosa y representantes de la subtribu Cranichidinae al que pertenece P. multiflora, presentan mesófilo homogéneo.

Myrosmodes paludosa presenta la mayor proporción de parénquima en empalizada, un carácter xeromorfo importante ya que el alto porcentaje de este tejido se fundamenta en el hecho de que el desarrollo es estimulado por la exposición a luz. Sin embargo, A. paleacea y $P$. multiflora por crecer en sitios protegidos (como es el caso del bosque de Polylepis sericea) presentan el parénquima en empalizada de una a dos corridas de células, el menor número de capas de parénquima en empalizada podría evitar la presencia de muchas paredes transversales que interrumpirían y reflejarían el flujo de luz (Esau 1976, Lindorf 1991).

Los haces colaterales cerrados son características propias en monocotiledóneas (Arber 1925, Esau 1976, Tomlinson 1969). Vaina vascular parenquimática de una corrida de células con almidón, que según Alexandrov (1926), Rhoades \& Carvalho (1944), es formado por los cloroplastos durante la fotosíntesis (Esau 1976). Esta vaina parenquimática también la consideran, en monocotiledóneas, una envoltura uniestrata comparable a la endodermis (Lindorf et al. 1991).

El pedúnculo se presenta lateral para A. paleacea y M. paludosa mientras que terminal para $P$. multiflora, con espigas de flores pequeñas no-resupinadas, características representativas para las subtribus a las cuales pertenecen (Dressler 1993).

Anatómicamente, se evidencia la epidermis de una corrida de células engrosadas y cutícula engrosada, este carácter favorece la protección contra los efectos del viento y daños mecánicos y se consideran que son características xeromorfas. Stern et al. (1993b) señalan en su estudio que las especies presentan células epidérmicas con cutículas finas, esta diferencia puede ser debida a que las especies aquí estudiadas crecen en el páramo que es un medio con condiciones más extremas. 
Se observa una corteza formada por un tejido parenquimático que puede actuar como reservante de agua y solutos y un tejido esclerénquimático que está relacionado con una función mecánica y posiblemente como reservorio hídrico, de almidón o aceites y proporciona al tallo resistencia frente a diferentes excesos evitando que sufran daño (Esau 1976, Lindorf et al. 1991, Rojas 2000); observaciones similares fueron realizadas por Stern et al. (1993b) para los representantes de la subtribu Diceratosteleae pero no así para los de Chranichideae, estas diferencias pueden ser debidas también al hecho de que entre el gran número de especies estudiados por los autores citados no se encontraba ninguno de los páramos. Los haces vasculares colaterales cerrados característicos de monocotiledóneas están dispuestos ya sea en una atactostela en $A$. paleacea y P.multiflora y en 2 anillos concéntricos en
M. paludosa, en todos los casos el anillo más externo parece estar asociado al anillo esclerenquimático más interno de la corteza. Esta observación coincide con Stern et al., (1993b), posiblemente se trate de una zona generatriz de haces que se observa también en otras orquídeas de páramo como Alteinstenia fimbriata Kunth y Gomphichis bogotensis Renz (observación personal).

Lisis y/o rompimiento mecánico en la médula parenquimática de A. paleacea y $P$. multiflora. En muchos tallos la médula se destruye en forma parcial o total durante el crecimiento, según Lindorf et al. (1991) esto ocurre debido a la expansión radial y según Flores (1999) el colapso de debe a la presión que ejercen los nuevos tejidos.

El análisis de las plantas estudiadas indica la presencia de características xeromórficas, que pueden interpretarse como adaptativas al páramo.

\section{Claves para la separación de los representantes de la tribu Cranichideae de la Estación Loma Redonda del teleférico de Mérida-Venezuela con base a caracteres morfológicos y anatómicos}

\section{Clave Morfológica}

1 Hoja peciolada. Roseta basal creciendo separada del suelo. Brácteas foliosas en el pedúnculo. Flores amarillas con rayas rojas P. multiflora

1' Hoja sésil. Roseta basal creciendo adosada al suelo. Brácteas hialinas en el pedúnculo. Flores blancas....... 2

2 Hoja elíptico-lanceolada, superficie adaxial opaca. Pedúnculo con desarrollo de entrenudos largos.

A. palacea

2' Hoja elíptico oblanceolada hasta aovada, superficie adaxial brillante. Pedúnculo con desarrollo de entrenudos cortos M. paludosa

\section{Clave Anatómica}

1 Hoja con la epidermis de la superficie adaxial no papilosa, cutícula lisa, la empalizada de 4-5 corrida de células. Corteza parenquimática de la raíz de 16-18 corrida de células, corteza esclerenquimática del pedúnculo de 3 corrida de células. M. paludosa

1' Hoja con la epidermis de la superficie adaxial papilosa, cutícula estriada, la empalizada de 1-2 corrida de células. Corteza parenquimática de la raíz de 13-15 corrida de células, corteza esclerenquimática del pedúnculo de 5-6 corrida de células.

2 Hoja hipostomática. Polos xilemáticos de la raíz de 10-16, corteza parenquimática del pedúnculo de 1-2 corrida de células. A. paleacea

2' Hoja anfiestomática. Polos xilemáticos de la raíz de aproximadamente 8 , corteza parenquimática del pedúnculo de 3-4 corrida de células.

Agradecimientos. Este trabajo se realizó en el Laboratorio de Anatomía Vegetal del Centro Jardín Botánico de la Facultad de Ciencias de la Universidad de los Andes. Los autores desean expresar su agradecimiento al CDCHT por el financiamiento parcial a través del proyecto $\mathrm{N}^{\circ} \mathrm{C}-119503$. A la gerencia del
Teleférico de Mérida por permitir el ascenso a la estación Loma Redonda y al personal por facilitar el traslado. También agradecen al profesor Javier Estrada, y de manera especial a la Lic. Josefina Peraza y al Per. For. Giusseppe Adamo por su valiosa colaboración durante su ejecución. 


\section{LiterATURA CITADA}

Álvarez, A. 2005. Phylogenetics of Prescottiinae Dressler y systematics of Gomphichis lindl. (Orchidaceae). A dissertation submitted to the Graduate Faculty in Biology in partial fulfillment of the requirements for the degree of Doctor of Philosophy, The City University of New York.

Arber, A. 1961[1925]. Monocotiledons. A morphological study. Cambridge Univ Press, Cambridge. pp 258.

Arditti, J. 1992. Fundamentals of orchid biology. John Wiley \& Sons, New York.

Carnevali, G., G. Gerlach \& G. Romero. 2005. Orchidaceae. Pp. 753-789 in: O. Hocke, P.E. Berry \& O. Huber (eds.), Nuevo Catálogo de la Flora Vascular de Venezuela. Fundación Instituto Botánico de Venezuela Dr. Tobías Lasser. Caracas-Venezuela.

Chase, M.W. 2005. Classification of orchidaceae in the age of dna data. The Board of Trustees of the Royal Botanic Gardens, Kew 2005.

Colmenares-Arteaga, M., F. Rada \& R. Luque. 2005. Anatomía foliar de Polylepis sericea Wedd. (Rosaceae) a dos altitudes en los Altos Andes Venezolanos. Plantula 3(3): 141-148.

Cribb, P. 2003. Subtribe Cranichidinae, Description and artificial key to the genera. Pp 23-24 in: A.C. Pridgeon, P.J. Cribb, M.W. Chase \& F.N. Rasmussen (eds.), Genera Orchidaceaerum 3. Oxford University Press, Oxford.

Dahlgren, R.M.T., H.T. Clifford \& P.F. Yeo. 1985. The families of the Monocotyledons. Springer-Verlag, Berlin.

Dengler, N.G. 1994. The influence of light on leaf development. pp. 100-136 in: M. Iqbal (ed.), Growth patterns in vascular plants. Dioscorides Press. Portland.

Dressler, R.L. 1993. Phylogeny and classification of the orchid family. Dioscorides Press, Portland.

Dueñas, G.H.C. \& J.L. Fernández-Alonso. 2009. Sinopsis de la subfamilia Spiranthoideae (Orchidaceae). Rev. Acad. Colomb. Cienc. 33(127): 157-182.

Eames, A.J. \& L.H. MacDaniels, 1947. An introduction to plant anatomy. McGraw-Hill Book Company, New York.

Ely, F. 1996. Anatomía foliar de Melastomateceae a lo largo de un gradiente altitudinal en el Parque Nacional Sierra Nevada de Mérida. Tesis de Grado de Licenciatura. Facultad de Ciencias, Universidad de los Andes. Mérida-Venezuela.

Esau, K. 1976. Plant anatomy. Barcelona-España.

Escalona, A. 1999. Anatomía foliar y del xilema de arbustos con aspecto de Gimnosperma en el Parque Nacional Sierra Nevada. Mérida-Venezuela. Tesis de grado de Licenciatura. Facultad de Ciencias, Universidad de los Andes. Mérida-Venezuela.
Estelita, M.E.T. \& W. Handro. 1983. Leaf ultrastructure in species of Gomphrena and Pfaffia (Amaranthaceae). Can. J. Bot. 62: 812-817.

Fahn, A. 1967. Plant anatomy. 4ta ed. (1979). Pergamom Press. Canadá.

Fahn, A. 1974. Plant anatomy. 2da ed. Ediciones H. Blume. Madrid-España.

Figueroa, C., G.A. Salazar, H.A. Zavaleta \& E.M. Engleman. 2007. Root character evolution and systematics in Cranichidinae, Prescottiinae and Spiranthinae (Orchidaceae, Cranichideae). Ann. Bot. 1 -12.

Flores, E.V. 1999. La planta. Estructura y función. Vol. II. Libro Universitario Regional. Costa Rica.

Gaulhofer, K. 1908. Die perzeption der Lichtrichtung im Laubblätte mit Hilfe der Randtüpfel, Randspalten und der windschiefen Radialwände. Sber. Akad. Wiss. Wien (Math Nat. K.) 1(117): 153-190.

Garay, L.A. 1978. Orchidaceae: Cypripedioideae, Orchidoideae, Neottiodeae. Pp. 3-304 in: Harling, G. \& B. Sparre (Eds.). Flora of Ecuador 9.

Garay, L.A. 1963. Evolutionary significance of geographical distribution of orchids. Proc. 4th Int Orchid Conference, Singapore: 170-187.

García, M.C.LL. 2004. Crecimiento, morfoanatomía radical, acumulación de solutos orgánicos y relaciones iónicas en dos genotipos de caña de azúcar (Sacharum spp) con tolerancia salina contrastante. Tesis de Doctorado. Facultad de Agronomía, Universidad Central de Venezuela.

Haberlandt, G. 1990[1884]. Physiological plant anatomy today \& tomorrow's. XVII . Printers \& Ruble, New Delhi.

Hose, E., D.T. Clarkson, E. Steudle, L. Shreiber, \& W. Hartung, 2001. The exodermis: a variable apoplastic barrier. J. Experim. Bot. 52: 2245-2264.

Jenik, J. 1978. Roots and root systems in tropical trees: morphological and ecological aspects. In: Tomlinson, P.B. \& M.H. Zimmermann (Eds.), Tropical trees as living systems. Cambridge University Press, Cambridge.

Johanssen, D.A. 1940. Botanical microtchnique. Mc Graw Hill Book. New York.

Lasser, T. 1969. Flora de Venezuela. Vol. XV. I parte. Instituto Botánico. Dirección de Recursos Naturales Renovables. Ministerio de Agricultura y Cría. Caracas.

Lindorf, H., L. Parisca, \& P. Rodríguez. 1991. Botánica, clasificación, estructura, reproducción. Universidad Central de Venezuela. Ediciones de la Biblioteca. Caracas-Venezuela.

Luque, R. A. 2004. Estructura primaria del sistema radical de Coespeletia Cuatrec. Interciencia 29(1): 13-18.

Luque, R.A. 2000. El sistema radical de Coespeletia Cuatrec. del páramo de Piedras Blancas, Mérida. 
Trabajo de Ascenso a la categoría de Prof. Asociado. Universidad de los Andes.

Luque, R.A., N.L. Menezes, \& J. Semir. 1999. Anatomía foliar de Lychnophora Mart. (Vernonieae: Asteraceae). Plantula 2(3): 141-152.

Luque R.A., H. Sousa, \& J. Kraus. 1996. Métodos de coloraçao de Roeser (1972) modificado e Kropp (1972) visando a substitução do azul de astra por azul de alcião 8 GS ou 8GX. Acta Bot. Brasil. (10): 199-212.

Monasterio, M. \& S. Reyes. 1980. Diversidad ambiental y variaciones en la vegetación de los páramos de los Andes Venezolanos. Pp. 47-92 in: Monasterio, M. (ed.), Estúdios ecológicos de los páramos andinos. Mérida: Universidad de Los Andes

Perumalla, C.J. \& C.A. Peterson. 1986. Deposition of Casparian bends and suberin lemellae in the exodermis and endodermis of young corn and onion roots. Canad. J. Bot. 64(7-9):1873-1878.

Petit, A.P.M. 1984. Variación altitudinal de la vegetación en los Andes Venezolanos. Tesis de grado. Facultad de Ciencias Forestales y Geografía, Universidad de los Andes. Mérida-Venezuela.

Porembski S. \& W. Barthlott. 1988. Velamen radicum micromorphology and classification of Orchidaceae. Nordic J. Bot. 8: 117-137.

Pridgeon A.M, P.J. Cribb, M.W. Chase, F.N. Rasmussen, eds. 2003. Genera Orchidacearum. Vol. 3. Orchidoideae. Part 2. Vanilloideae. Oxford University Press, Oxford.

Pyykkö, M. 1966. The leaf anatomy of East Patagonia xeromorphic plants. Ann. Bot. Fenn. 3: 453-622.

Rada. F., G. Goldstein, A. Azócar, \& F. Meinzer, 1985. Freezing avoidance in Andean giant rosette plants. P1. Cell Environ. 8: 501-507.

Rasmussen, H.N. \& D.F. Whigham, 1998. The underground phase: a special challenge in studies of terrestrial orchid populations. Bot. J. Linn. Soc.126: 49- 64.

Ricardi, M.H. 1988. Familias de monocotiledóneas Venezolanas. 1ra Edición. Consejo de Publicaciones de la Universidad de los Andes. Mérida-Venezuela.

Rojas. M. 2002. Anatomía en órganos vegetativos y en el tallo floral de los géneros Orthrosanthus S. y Sisyrinchium L. (Iridaceae) que crecen en el Parque
Nacional Sierra Nevada de Mérida. Tesis de Grado de Licenciatura. Facultad de Ciencias, Universidad de los Andes. Mérida-Venezuela.

Roth, I. 1990. Leaf structure of a Venezuelan cloud forest in relation to the microclimate. Encyclopedia of Plan Anatomy. Gebrüder Bontraeger. Berlin. Stuttgart.

Roth, I. 1991. Anatomía de las plantas superiores. Universidad Central de Venezuela. Ediciones de la Biblioteca.Caracas.

Saas, J.E. 1958. Botanical Microtechnique, 3ra edición. Editorial Lona State Collage Press. New York.

Salazar, G.A., L.I. Cabrera, S. Madriñán \& M.W. Chase. 2009. Phylogenetic relationships of Cranichidinae and Prescottiinae (Orchidaceae, Cranichideae) inferred from plastid and nuclear DNA sequences. Ann. Bot.104: 403-416.

Stern, W.L., H.C. Aldrich, L.M. Mcdowell, M.W. Morris \& A.M. Pridgeon. 1993a. Amyloplasts from cortical root cells of Spiranthoideae (Orchidaceae). Protoplasma 172: 49-55.

Stern, W.L., M.W. Morris, W.S. Judd, A.M. Pridgeon, \& R.L. Dressler. 1993b. Comparative vegetative anatomy and systematic of Spiranthoideae (Orchidaceae). Bot. J. Linn. Soc. 113: 161-197.

Tomlinson, P. 1969. Anatomy of the Monocotyledons. Vol. III. Pp. 92-126 en: Metcalfe, C. (ed.), CommelinalesZingiberales. Clarendon press. Oxford.

Torres, F. 1998. Anatomía foliar en Coespeletia Cuatrec del páramo de Piedras Blancas. Plan estructural básico y sus modificaciones. Trabajo de Ascenso. Universidad de los Andes. Mérida-Venezuela.

Torres, F.R., J.C. Gaviria \& J. Peraza. 1996. Anatomía foliar de Oritrophium (H.B.K.) Cuatrec. del páramo de Sierra Nevada, Estación Loma Redonda. Plantula 1(1): 65-73.

Trujillo, D. \& A.R. Delgado. 2011. Aa from lomas format ions. A New Orchidaceae record from the desert coast of Peru. Lankesteriana 11(1): 33-38.

Van Fleet, D.S. 1961. Histochemistry and function of the endodermis. Bot. Rev. 27(2): 165-219.

Wilkinson, H. 1979. The plant surface (mainly leaf): stoma. 2da ed. Vol. I. in: Metcalfe, C. \& L. Chalk, (eds.), Anatomy of the dicotyledons. Clarendon Press. Oxford. 